\title{
Stress-Induced Dinoflagellate Bioluminescence at the Single Cell Level
}

\author{
Maziyar Jalaal@ ${ }^{1}$ Nico Schramma $\odot,{ }^{1,2}$ Antoine Dode $\odot,{ }^{1,3}$ Hélène de Maleprade $\odot,{ }^{1}$ \\ Christophe Raufaste $\oplus^{1,4}$ and Raymond E. Goldstein $\circledast^{1, *}$ \\ ${ }^{1}$ Department of Applied Mathematics and Theoretical Physics, University of Cambridge, Cambridge CB3 OWA, United Kingdom \\ ${ }^{2}$ Max Planck Institute for Dynamics and Self-Organization, 37077 Göttingen, Germany \\ ${ }^{3}$ LadHyX, UMR 7646 du CNRS, École polytechnique, 91120 Palaiseau, France \\ ${ }^{4}$ Université Côte d'Azur, CNRS, Institut de Physique de Nice, CNRS, 06100 Nice, France
}

(Received 18 March 2020; accepted 26 May 2020; published 6 July 2020)

\begin{abstract}
One of the characteristic features of many marine dinoflagellates is their bioluminescence, which lights up nighttime breaking waves or seawater sliced by a ship's prow. While the internal biochemistry of light production by these microorganisms is well established, the manner by which fluid shear or mechanical forces trigger bioluminescence is still poorly understood. We report controlled measurements of the relation between mechanical stress and light production at the single cell level, using high-speed imaging of micropipette-held cells of the marine dinoflagellate Pyrocystis lunula subjected to localized fluid flows or direct indentation. We find a viscoelastic response in which light intensity depends on both the amplitude and rate of deformation, consistent with the action of stretch-activated ion channels. A phenomenological model captures the experimental observations.
\end{abstract}

DOI: 10.1103/PhysRevLett.125.028102

Bioluminescence, the emission of light by living organisms, has been a source of commentary since ancient times [1], from Aristotle and Pliny the Elder, to Shakespeare and Darwin [2], who, like countless mariners before him, observed of the sea, “... every part of the surface, which during the day is seen as foam, now glowed with a pale light. The vessel drove before her bows two billows of liquid phosphorus, and in her wake she was followed by a milky train. As far as the eye reached, the crest of every wave was bright ..." The glow Darwin observed likely arose from bacteria or dinoflagellates, unicellular eukaryotes found worldwide in marine and freshwater environments.

Bioluminescence occurs in a large range of organisms, from fish to jellyfish, worms, fungi, and fireflies. While discussion continues regarding the ecological significance of luminescence [3], the internal process that produces light is now understood. In dinoflagellates [4], changes in intracellular calcium levels produce an action potential, opening voltage-gated proton channels in the membranes of organelles called scintillons, lowering the $\mathrm{pH}$ within them [5] and causing oxidation of the protein luciferin, catalyzed by luciferase. Far less clear is the mechanism by which fluid motion triggers bioluminescence.

Published by the American Physical Society under the terms of the Creative Commons Attribution 4.0 International license. Further distribution of this work must maintain attribution to the author(s) and the published article's title, journal citation, and DOI.
Early experiments on light emission utilizing unquantified fluid stirring or bubbling [6] were superseded over the past two decades by studies in the concentric cylinder geometry of Couette flow [7,8] and macroscopic contracting flows $[9,10]$. Subsequent experiments explored luminescence by cells carried by flow against microfluidic barriers [11] or subjected to the localized forces of an atomic force microscope [12]. From these have come estimates of the stress needed to trigger light production. Indeed, dinoflagellates can serve as probes of shear in fluid flows [7,9,13-16]. At the molecular level, biochemical interventions have suggested a role for stretch-activated ion channels [17] — known to feature prominently in touch sensation [18]—leading to the hypothesis that fluid motion

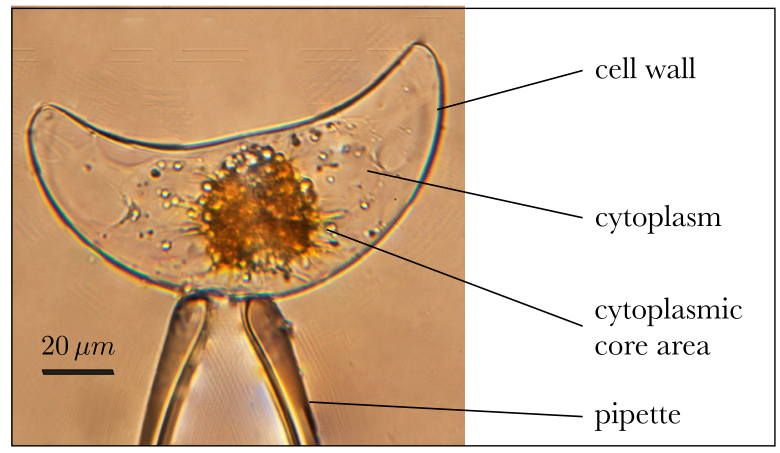

FIG. 1. The unicellular marine dinoflagellate Pyrocystis lunula, held on a glass micropipette. Chloroplasts (yellow-brown) are in the cytoplasmic core at night and the crescent-shaped cell wall encloses the cell. 

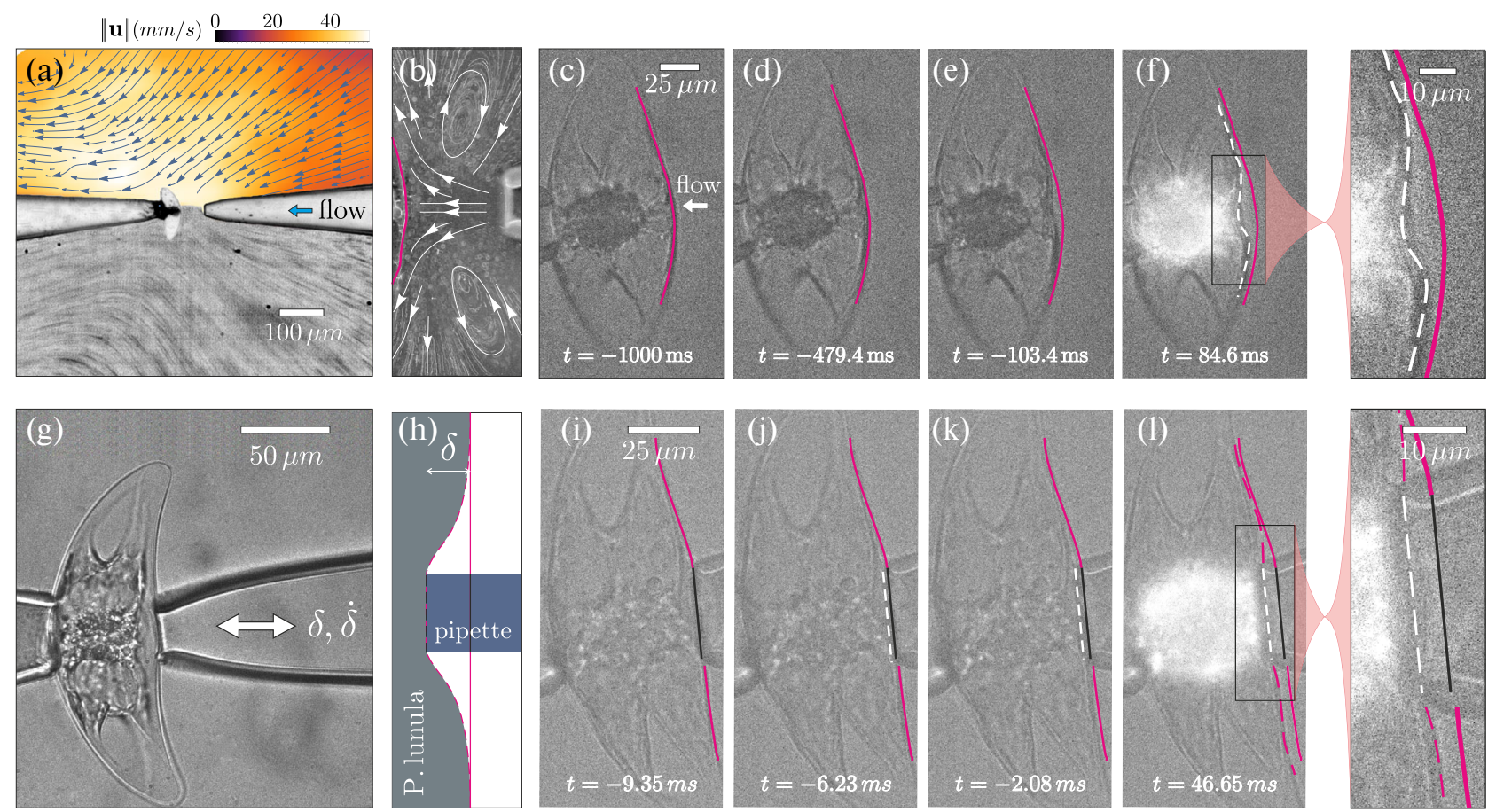

FIG. 2. Light production by $P$. Lunula under fluid and mechanical stimulation. (a) Stimulation by fluid flow; color map in upper half indicates flow speed, lower half is a streak image of tracers. (b) Particle tracking of flow lines near cell surface. (c)-(f) Cell deformation due to flow and consequent light production. (g),(h) Second protocol, in which a cell is deformed under direct contact by a second pipette $\left(\delta_{f}=10, \dot{\delta}=76 \mu \mathrm{m} / \mathrm{s}\right)$. (i)-(l) Light production triggered by mechanical deformation. Indicated times are with respect to start of light emission.

stretches cellular membranes, forcing channels open and starting the biochemical cascade that produces light.

As a first step toward an in-depth test of this mechanism, we study luminescence of single cells of the dinoflagellate Pyrocystis lunula (Fig. 1) induced by precise mechanical stimulation. The cellular response is found to be "viscoelastic"; it depends not only on the amplitude of cell wall deformation, but also on its rate. A phenomenological model linking this behavior to light production provides a quantitative account of these observations.

$P$. lunula is an excellent organism for the study of bioluminescence because its large size $(\sim 40 \mu \mathrm{m}$ in diameter and $\sim 130 \mu \mathrm{m}$ in length), lack of motility as an adult, rigid cell wall, and negative buoyancy facilitate micromanipulation. Its transparency and featureless surface allow for high-resolution imaging. As model organisms, dinoflagellates have been studied from many complementary perspectives [19].

Cultures of $P$. lunula (Schütt) obtained from the Culture Collection of Algae and Protozoa [20] were grown in L1 medium [21] at $20^{\circ} \mathrm{C}$ in an incubator on a $12 \mathrm{~h} / 12 \mathrm{~h} \mathrm{light/}$ dark cycle. The blue $(\sim 475 \mathrm{~nm})$ bioluminescence of $P$. lunula is under circadian regulation [22,23] and occurs only during the night. All experiments were performed between hours 3 and 5 of the nocturnal phase. A CMOS camera (Prime 95B, Photometrics) imaged cells through a Nikon $63 \times$ water-immersion objective on a Nikon TE2000 inverted microscope. Cells were kept in a $500 \mu \mathrm{L}$ chamber that allows access by two antiparallel micropipettes held on micromanipulators (Patchstar, Scientifica, UK) (see Supplemental Material [24]) and kept undisturbed for several hours prior to stimulation. Upon aspiration on the first pipette, cells typically flash once [25]. Care was taken to achieve consistent positioning of cells for uniformity of light measurements (Supplemental Material, Video 1 [24]).

The second pipette applies mechanical stimulation in either of two protocols. The first directs a submerged jet of growth medium at the cell, controlled by a syringe pump (PHD2000, Harvard Apparatus) and characterized using particle image velocimetry (PIV) and particle tracking [Figs. 2(a)-2(f)]. Typical flow rates through the micropipette were $\sim 1 \mathrm{ml} / \mathrm{h}$, exiting a tip of radius $\sim 10 \mu \mathrm{m}$, yielding speeds $U=0.1-1 \mathrm{~m} / \mathrm{s}$. With $\nu=\eta / \rho=1 \mathrm{~mm}^{2} / \mathrm{s}$ the kinematic viscosity of water, where $\rho$ is its density, and $\ell \sim 0.02 \mathrm{~mm}$ the minor radius of the organism, the Reynolds number is $\operatorname{Re}=U \ell / \nu \sim 2-20$, consistent with studies in macroscopic flows $[7,9,10]$ that used the apparatus scale (millimeter) for reference. In the second protocol, a cell is held between the two pipettes, and deformation is imposed by displacement of the second. Using the micromanipulators and a computer-controlled translation stage (DDS220/M, Thorlabs), the deformation $\delta$ and rate $\dot{\delta}$ could be independently varied [Figs. 2(g)-2(l)]. 

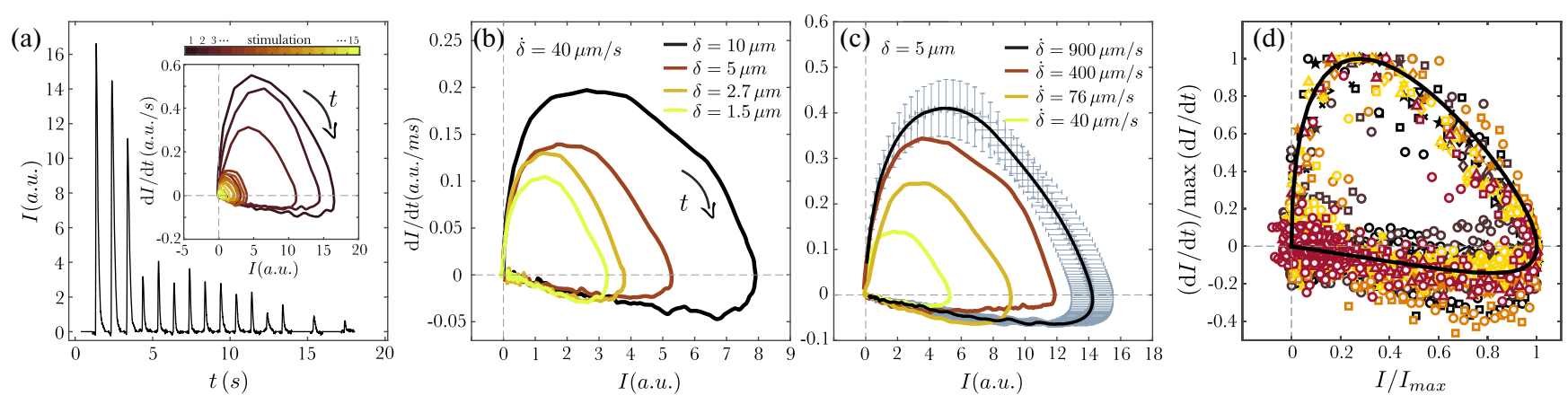

FIG. 3. Dynamics of light production following mechanical stimulation. (a) Response of a cell to repeated deformation with $\delta_{f}=$ $10 \mu \mathrm{m}$ and $\dot{\delta}=76 \mu \mathrm{m} / \mathrm{s}$. Inset: Loops in $I-d I / d t$ plane for successive flashes (black to yellow). (b) Loops at fixed $\dot{\delta}$ and varying $\delta_{f}$ for the first flash under given parameters. (c) As in (b), but for fixed $\delta_{f}$ and varying $\dot{\delta}$. Standard errors are shown for outermost data. (d) Master plot of data from 25 (out of a total of 164) first flashes, normalized by maximum intensities and rates. Circles (squares) are data in (b) (c). Black curve is result of model in (1) and (3).

A key observation within the first protocol is that cells do not flash unless the imposed fluid pressure is high enough to deform sufficiently the cell wall [Fig. 2(f)]. For these jet flows, with an intermediate speed of $0.3 \mathrm{~m} / \mathrm{s}$, the normal stress $\Sigma_{f} \sim$ $\rho U^{2}$ is $\sim 10^{2} \mathrm{~Pa}$, several orders of magnitude larger than in macroscopic experiments [7-10]. From Fig. 2(f), we see that deformations have strains $\varepsilon=\delta / \ell \sim 0.1-1$ with a lateral scale $\xi \sim 30 \mu \mathrm{m}$, and we estimate the fluid force exerted at the site of deformation as $F_{f} \sim \Sigma_{f} \xi^{2} \sim 0.01-0.1 \mu \mathrm{N}$. More quantitatively, using PIV of the flow field and finite-element calculations of the flow from a pipette [24], we find from study of 35 cells that the threshold for luminescence is broadly distributed, with a peak at $0.10 \pm 0.02 \mu \mathrm{N}$. Those studies also show that the normal forces are typically a factor of 3 larger than the shear stress on the cell wall.

It is not clear a priori whether the deformations in Figs. 2(c)-2(f) are resisted by the cell wall alone or also by the cytoplasm. The wall has a tough outer layer above a region of cellulose fibrils [26-28], with a thickness $d \sim 200-400 \mathrm{~nm}:$ AFM studies [12] show a Young's modulus $E \sim 1 \mathrm{MPa}$. During asexual reproduction, the cellular contents pull away from the wall and eventually exit it through a hole, leaving behind a rigid shell with the characteristic crescent moon shape [29]. Thus, the wall is not only imprinted with that shape, but is much more rigid than the plasma membrane and significantly more rigid than the cytoplasm [12].

Deformations of such curved structures induced by localized forces involve bending and stretching of the wall. Taking the minor radius $\ell$ as the radius of curvature of the undeformed cell wall, a standard analysis [30] gives the indentation force $F \sim E d^{2} \delta / \ell$. Balancing this against the fluid force $\rho U^{2} \xi^{2}$ we find the strain $\varepsilon \sim\left(\rho U^{2} / E\right)(\xi / d)^{2}$. From the estimates above, we have $\rho U^{2} / E \sim 10^{-4}$ and $\xi / d \sim 50-100$, so $\varepsilon$ can reach unity, as observed.

In the natural marine setting and in laboratory studies of dilute suspensions, luminescence can arise purely from flow itself, without intercellular contact. Nevertheless, there are conceptual and methodological advantages to studying bioluminescence by mechanical contact, especially due to the natural compliance of cells aspirated by a single micropipette. Chief among these is the ability to control the deformation and deformation rate, which are the most natural variables for quantification of membrane stretching and bending. As seen in Figs. 2(i)-2(1), imposing deformations similar to those achieved with fluid flow also produces bioluminescence, highlighting the role of cell membrane deformation in mechanosensing.

In our protocol for deformations, $\delta$ is increased at a constant rate $\dot{\delta}$ for a time $t_{f}$ to a final value $\delta_{f}$ ("loading"), after which it was held fixed until any light production ceases, then returned to zero ("unloading"). We observe, generally, that if light is produced during loading, it is also produced during unloading. Experiments were performed for $\delta_{f} \in[1,10] \mu \mathrm{m}$ and $\dot{\delta} \in[10,900] \mu \mathrm{m} / \mathrm{s}$, with $6-8$ replicates (cells) for each data point. We repeated a given protocol on the same cell (with sufficient rest intervals in between) until the cell ceased bioluminescence. Reported values of light intensity $I(t)$ are integrated over the entire cell.

Figure 3(a) shows the light flashes from 15 stimulations of a single cell. With each deformation, $I(t)$ rises rapidly and then decays on a longer timescale. Apart from a decreasing overall magnitude with successive flashes, the shape of the signal remains nearly constant. The eventual loss of bioluminescence most likely arises from exhaustion of the luciferin pool [31]. The inset shows the corresponding phase portraits of the flashes in the $I-d I / d t$ plane, highlighting the similarity of successive signals.

Focusing on the very first flashes, experiments with different $\delta_{f}$ and $\dot{\delta}$ reveal the systematics of luminescence. Figures 3(b) and 3(c) show that, for a given rate, larger deformations produce more light, as do higher rates at a given deformation. Interestingly, the shape of the signals remains the same not only between different cells but also for different mechanical stimulations; normalizing the 

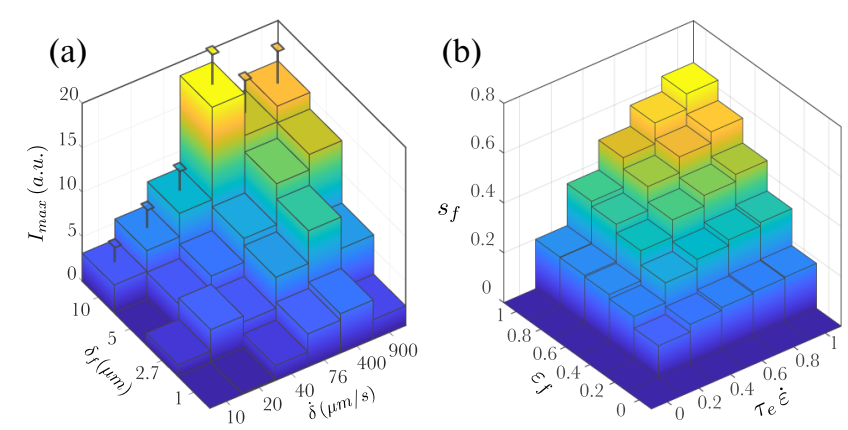

FIG. 4. Dependence of light production on deformation and rate. (a) Histogram of maximum intensity with standard errors for $\delta_{f}=10 \mu \mathrm{m}$. Note nonuniform grid. (b) Variation of signal strength $s_{f}$ predicted by phenomenological model, as a function of deformation and rate.

phase portraits by their maxima yields a universal signal shape [Fig. 3(d)]. Figure 4(a) summarizes all the results, showing the variation of maximum intensity (averaged over first flashes) as a function of $\delta_{f}$ and $\dot{\delta}$; luminescence is maximized when cells are highly deformed at high speed.

The influence of deformation and rate are suggestive of viscoelastic properties. At a phenomenological level, we thus consider a Maxwell-like model that relates the signal $s(t)$ that triggers light production to the strain $\varepsilon$,

$$
\dot{s}+\tau_{e}^{-1} s=\dot{\varepsilon},
$$

where $\tau_{e}$ is a relaxation time. For a given $\delta$, if the deformation timescale is much smaller than $\tau_{e}$, the membrane does not have time to rearrange (the large Deborah number regime in rheology), while for slow deformations the membrane has time to relax. As seen in Figs. 2(i)-2(1) and Supplemental Material Videos 2 and 3 [24], bioluminescence occurs during loading, a feature that suggests $\tau_{e}$ is comparable to the flash rise time. Integrating (1) up to $t_{f}$, we obtain the signal $s_{f}$ at the end of loading in terms of the final strain $\varepsilon_{f} \equiv \delta_{f} / \ell$ and scaled strain rate $\dot{\varepsilon} \tau_{e}$,

$$
s_{f}=\dot{\varepsilon} \tau_{e}\left(1-\mathrm{e}^{-\varepsilon_{f} / \dot{\varepsilon} \tau_{e}}\right) .
$$

Figure 4(b) shows that the peak response occurs when both the final strain and strain rate are large, as observed experimentally. The linear relationship between $s$ and $\varepsilon$ embodied in (1) cannot be valid at large strains or strain rates; eventually, the signal must saturate when all available channels open. This is consistent with the data in Fig. 4(a) at the highest rates, where experimentally $\varepsilon \sim 0.25$.

Although light production is triggered internally by an action potential-which arises from nonlinear, excitable dynamics - analysis of the flashes [24] indicates a time course much like that of two coupled capacitors charging and discharging on different timescales. Such linear dynamics have figured in a variety of contexts, including calcium oscillations [32], bacterial chemotaxis [33], and algal phototaxis [34], and take the form of coupled equations for the observable (here, the light intensity $I$ ), which reacts to the signal $s$ on a short time $\tau_{r}$, and the hidden biochemical process $h$, which resets the system on a longer time $\tau_{a}$. For light triggered by stretch-activated ion channels, the signal $s$ might be the influx of calcium resulting from the opening of channels. Adopting units in which $I, h$, and $s$ are dimensionless, the simplest model is

$$
\begin{aligned}
\tau_{r} \dot{I} & =s-h-I, \\
\tau_{a} \dot{h} & =s-h .
\end{aligned}
$$

Starting from the fixed point $(I=0, h=0)$ for $s=0$, if the signal is turned on abruptly then $I$ will respond on a timescale $\tau_{r}$, exponentially approaching $s-h \simeq s$. Then, as $h$ evolves toward $s$ over the longer adaptation timescale $\tau_{a}$, $I$ will relax toward $s-h \simeq 0$, completing a flash. It follows from (3) that a discontinuous initial $s$ creates a discontinuous $\dot{I}$, whereas the loops in Fig. 3 show smooth behavior in that early regime $(I, \dot{I} \gtrsim 0)$; this smoothing arises directly from the Maxwell model (1) for the signal. The parsimony of the linear model (3) comes at a cost, for it fails at very high ramp rates when $\dot{\varepsilon}$ switches to zero within the flash period and both $s$ and $I$ would adjust accordingly, contrary to observations. In a more complex, excitable model, the flash, once triggered, would thereafter be insensitive to the signal.

As the entire system (1) and (3) is linear, it can be solved exactly [24], thus enabling a global fit to the parameters. We compare the theoretical results with the normalized experimental data in Fig. 3(d), where we see good agreement with the common loop structure. From the fits across all data, we find common timescales $\tau_{e} \approx 0.027$, $\tau_{r} \approx 0.012$, and $\tau_{a} \approx 0.14 \mathrm{~s}$, the last of which is comparable to the pulse decay time found in earlier experiments with mechanical stimulation [25] and can be read off directly from the late-time dynamics of the loops in Figs. 3(b) and 3(c), where $\dot{I} \sim-I / \tau_{a}$ [24]. These values suggest comparable timescales of membrane-channel viscoelasticity and biochemical actuation, both much shorter than the decay of light flashes.

With the results described here, the generation of bioluminescence has now been explored with techniques ranging from atomic force microscope cantilevers, with attached microspheres indenting cells in highly localized areas, to fluid jets and micropipette indentation on intermediate length scales, and finally to macroscopic flows that produce stresses across the entire cell body. Figure 5 considers all of these experiments together, organized by the perturbative stress $\Sigma$ found necessary to produce light and the area $\mathcal{A} \equiv \xi^{2}$ over which that stress was applied. We see a clear trend: the smaller the perturbation area, the larger the force required. This result suggests that the 


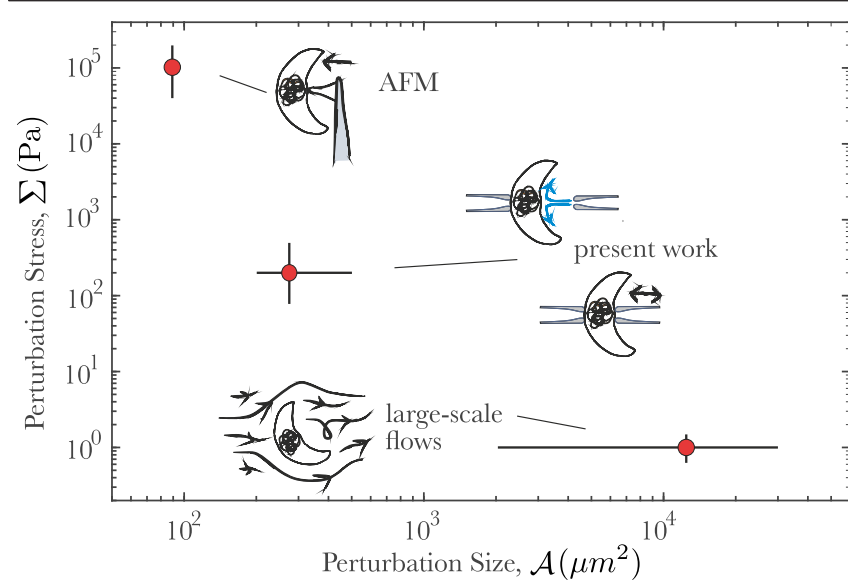

FIG. 5. Perturbation stress versus perturbation area for three kinds of experiments on $P$. lunula. Atomic force measurements are from [12], while macroscopic measurements are performed with a Taylor-Couette flow [8].

production of a given amount of light, through the probabilistic opening of stretch-activated ion channels, can be achieved through the action of many channels weakly recruited or a small number strongly recruited. With an eye toward connecting the present results to the familiar marine context of light production, it is thus of interest to understand more quantitatively the distribution of forces over the entire cell body in strong shear flows [35] and which components of those forces (normal or tangential) activate ion channels to produce light. Likewise, the possible ecological significance of the great range of possible excitation scales illustrated in Fig. 5 remains to be explored.

We are grateful to Michael I. Latz for invaluable assistance at an early stage of this work, particularly with regard to culturing dinoflagellates, and thank Adrian Barbrook, Martin Chalfie, Michael Gomez, Tulle Hazelrigg, Chris Howe, Caroline Kemp, Eric Lauga, Benjamin Mauroy, Carola Seyfert, and Albane Théry for important discussions. This work was supported in part by a Wellcome Trust Interdisciplinary Fellowship (MJ), the Gordon and Betty Moore Foundation (Grant No. 7523) and the Schlumberger Chair Fund (REG). C. R. acknowledges support by the French government, through the UCA ${ }^{\mathrm{JEDI}}$ Investments in the Future project of the National Research Agency (ANR) (ANR-15-IDEX-01).

*R.E.Goldstein@damtp.cam.ac.uk

[1] E. N. Harvey, The Nature of Animal Light (J. B. Lippincott Company, Philadelphia, 1920).

[2] C. Darwin, in Journal of Researches into the Geology and Natural History of the Various Countries Visited by H.M.S. Beagle, Under the Command of Captain Fitzroy, R.N. from 1832 to 1836 (Henry Colburn, London, 1839), p. 191.

[3] S. H. D. Haddock, M. A. Moline, and J. F. Case, Bioluminescence in the sea, Annu. Rev. Mar. Sci. 2, 443 (2010).
[4] T. Wilson and W. J. Hastings, Bioluminescence, Annu. Rev. Cell Dev. Biol. 14, 197 (1998); M. Valiadi and D. Iglesias-Rodriguez, Understanding bioluminescence in dinoflagellates-How far have we come?, Microorganisms 1, 3 (2013).

[5] M. Fogel and J. W. Hastings, Bioluminescence: Mechanism and mode of control of scintillon activity, Proc. Natl. Acad. Sci. U.S.A. 69, 690 (1972).

[6] W. H. Biggley, E. Swift, R. J. Buchanan, and H. H. Seliger, Stimulable and spontaneous bioluminescence in the marine dinoflagellates, Pyrodinium bahamense, Gonyaulax polyedra, and Pyrocystis lunula, J. Gen. Physiol. 54, 96 (1969); G. B. Deane, M. D. Stokes, and M. I. Latz, Bubble stimulation efficiency of dinoflagellate bioluminescence, Luminescence 31, 270 (2016).

[7] M. I. Latz, J.F. Case, and R. L. Gran, Excitation of bioluminescence by laminar fluid shear associated with simple Couette flow, Limnol. Oceanogr. 39, 1424 (1994); E. M. Maldonado and M. I. Latz, Shear-stress dependence of dinoflagellate bioluminescence, Biol. Bull. 212, 242 (2007).

[8] A.-S. Cussatlegras and P. Le Gal, Variability in the bioluminescence response of the dinoflagellate Pyrocystis lunula, J. Exp. Mar. Biol. Ecol. 343, 74 (2007).

[9] M. I. Latz, J. Rohr, and J. Hoyt, A novel flow visualization technique using bioluminescent marine plankton. I. Laboratory studies, IEEE J. Oceanic Eng. 20, 147 (1995).

[10] M. I. Latz, A. R. Juhl, A. M. Ahmed, S. E. Elghobashi, and J. Rohr, Hydrodynamic stimulation of dinoflagellate bioluminescence: A computational and experimental study, J. Exp. Biol. 207, 1941 (2004).

[11] M. I. Latz, M. Bovard, V. VanDelinder, E. Segre, J. Rohr, and A. Groisman, Bioluminescent response of individual dinoflagellate cells to hydrodynamic stress measured with millisecond resolution in a microfluidic device, J. Exp. Biol. 211, 2865 (2008).

[12] B. Tesson and M. I. Latz, Mechanosensitivity of a rapid bioluminescence reporter system assessed by atomic force microscopy, Biophys. J. 108, 1341 (2015).

[13] J. Rohr, J. Allen, J. Losee, and M. I. Latz, The use of bioluminescence as a flow diagnostic, Phys. Lett. A 228, 408 (1997).

[14] E. Foti, C. Faraci, R. Foti, and G. Bonanno, On the use of bioluminescence for estimating shear stresses over a rippled seabed, Meccanica 45, 881 (2010).

[15] J. Hauslage, V. Cevik, and R. Hemmersbach, Pyrocystis noctiluca represents an excellent bioassay for shear forces induced in ground-based microgravity simulators (clinostat and random positioning machine), npj Microgravity 3, 12 (2017).

[16] G. B. Deane and M. D. Stokes, A quantitative model for flow-induced bioluminescence in dinoflagellates, J. Theor. Biol. 237, 147 (2005).

[17] K. Jin, J. C. Klima, G. Deane, M. D. Stokes, and M. I. Latz, Pharmacological investigation of the bioluminescence signaling pathway of the dinoflagellat Lingulodinium polyedrum: Evidence for the role of stretch-activated ion channels, J. Phycol. 49, 733 (2013).

[18] C. Kung, A possible unifying principle for mechanosensation, Nature (London) 436, 647 (2005). 
[19] J. D. Hackett, D. M. Anderson, D. L. Erdner, and D. Bhattacharya, Dinoflagellates: A remarkable evolutionary experiment, Am. J. Bot. 91, 1523 (2004); C. Fajardo, F. Amil-Ruiz, C. Fuentes-Almagro, M. De Donato, G. Martinez-Rodriguez, A. Escobar-Niño, R. Carrasco, J. Miguel Mancera, and F. Javier Fernandez-Acero, An "omic" approach to Pyrocystis lunula: New insights related with this bioluminescent dinoflagellate, J. Proteomics 209, 103502 (2019).

[20] The Culture Collection of Algae and Protozoa (CCAP), https://www.ccap.ac.uk/index.htm.

[21] R. R. L. Guillard and P. E. Hargraves, Stichochrysis immobilis is a diatom, not a chrysophyte, Phycologia 32, 234 (1993).

[22] E. Swift and W. R. Taylor, Bioluminescence and chloroplast movement in the dinoflagellate Pyrocystis Lunula, J. Phycol. 3, 77 (1967).

[23] P. Colepicolo, T. Roenneberg, D. Morse, W. R. Taylor, and J. W. Hastings, Circadian regulation of bioluminescence in the dinoflagellate Pyrocystis Lunula, J. Phycol. 29, 173 (1993).

[24] See Supplemental Material at http://link.aps.org/ supplemental/10.1103/PhysRevLett.125.028102 for further experimental details and videos.

[25] E. A. Widder and J.F. Case, Two flash forms in the bioluminescent dinoflagellate, Pyrocystis fusiformis, J. Comp. Physiol. 143, 43 (1981).

[26] E. Swift and C. C. Remsen, The cell wall of Pyrocystis spp. (Dinococcales), J. Phycol. 6, 79 (1970).

[27] R. A. Fensome, F. J. R. Taylor, G. Norris, W. A. S. Sarjeant, D. I. Wharton, and G. L. Williams, A Classification of
Living and Fossil Dinoflagellates (Sheridan Press, Pennsylvania, 1993), Micropaleontology, Spec. Pub. No. 7.

[28] K. S. Seo and L. Fritz, Cell-wall morphology correlated with vertical migration in the non-motile marine dinoflagellate Pyrocystis noctiluca, Mar. Biol. 137, 589 (2000).

[29] E. Swift and E. G. Durbin, Similarities in the asexual reproduction of the oceanic dinoflagellates, Pyrocystis fusiformis, Pyrocystis lunula, and Pyrocystis noctiluca, J. Phycol. 7, 89 (1971).

[30] L. D. Landau and E. M. Lifshitz, in Theory of Elasticity, 3rd ed. (Elsevier, Amsterdam, 1986), p. 15.

[31] K. S. Seo and L. Fritz, Cell ultrastructural changes correlate with circadian rhythms in Pyrocystis lunula (Pyrrophyta), J. Phycol. 36, 351 (2000).

[32] A. Goldbeter, G. Dupont, and M. J. Berridge, Minimal model for signal-induced $\mathrm{Ca}^{2+}$ oscillations and for their frequency encoding through protein phosphorylation, Proc. Natl. Acad. Sci. U.S.A. 87, 1461 (1990).

[33] P. A. Spiro, J. S. Parkinson, and H. G. Othmer, A model of excitation and adaptation in bacterial chemotaxis, Proc. Natl. Acad. Sci. U.S.A. 94, 7263 (1997).

[34] K. Drescher, R. E. Goldstein, and I. Tuval, Fidelity of adaptive phototaxis, Proc. Natl. Acad. Sci. U.S.A. 107, 11171 (2010).

[35] R. Trans-Son-Tay, S. P. Sutera, G. I. Zahalak, and P. R. Rao, Membranes stresses and internal pressure in a red blood cell freely suspended in a shear flow, Biophys. J. 51, 915 (1987); A. Théry, M. Jalaal, E. Lauga, and R. E. Goldstein (to be published). 